\title{
The impact of a 16-week dietary intervention with prescribed amounts of whole-grain foods on subsequent, elective whole grain consumption
}

\author{
Iain A. Brownlee ${ }^{1}$, Sharron A. Kuznesof ${ }^{1}$, Carmel Moore $^{2}$, Susan A. Jebb ${ }^{2}$ and Chris J. Seal ${ }^{1 *}$ \\ ${ }^{1}$ The Human Nutrition Research Centre, School of Agriculture, Food and Rural Development, Newcastle University, \\ Newcastle upon Tyne NE1 7RU, UK \\ ${ }^{2}$ MRC Human Nutrition Research, Elsie Widdowson Laboratory, Fulbourn Road, Cambridge CB1 9NL, UK \\ (Submitted 30 May 2012 - Final revision received 20 September 2012 - Accepted 9 November 2012 - First published online 7 February 2013)
}

\section{Abstract}

Previous (mainly population-based) studies have suggested the health benefits of the elective, lifelong inclusion of whole-grain foods in the diet, forming the basis for public health recommendations to increase whole grain consumption. Currently, there is limited evidence to assess how public health recommendations can best result in longer-term improvements in dietary intake. The present study aimed to assess the impact of a previous 16-week whole-grain intervention on subsequent, elective whole grain consumption in free-living individuals. Participants completed a postal FFQ 1, 6 and 12 months after the end of the whole-grain intervention study period. This FFQ included inputs for whole-grain foods commonly consumed in the UK. Whole grain consumption was significantly higher (approximately doubled) in participants who had received whole-grain foods during the intervention $(P<0 \cdot 001)$ compared with the control group who did not receive whole-grain foods during the intervention. This increased whole grain consumption was lower than whole grain intake levels required by participants during the intervention period between 60 and $120 \mathrm{~g}$ whole grains/d. Aside from a significant increase $(P<0 \cdot 001)$ in NSP consumption compared with control participants (mean increase $2-3 \mathrm{~g} / \mathrm{d}$ ), there were no obvious improvements to the pattern of foods of the intervention group. The results of the present study suggest that a period of direct exposure to whole-grain foods in non-habitual whole-grain food consumers may benefit subsequent, elective dietary patterns of whole grain consumption. These findings may therefore aid the development of future strategies to increase whole grain consumption for public health and/or food industry professionals.

\section{Key words: Whole-grain foods: Dietary intervention: Post-intervention follow-up: Elective consumption}

Observational data suggest an association between increased whole grain consumption and decreased morbidity and mortality $^{(1-4)}$. While there is an increased drive from health professionals and some cereal-based food manufacturers for increased whole grain consumption at a population level ${ }^{(5)}$, data show that the vast majority of people in the USA ${ }^{(6)}$ and Denmark ${ }^{(7)}$ do not habitually consume the levels of whole-grain foods recommended by the US Department of Agriculture $^{(8)}$ or the Danish National Food Institute Technical University of Denmark ${ }^{(9)}$. Similar, or low, intake levels are reported for the UK population ${ }^{(10)}$, although there are no prescriptive guidelines in the UK currently.

Previous studies ${ }^{(11-13)}$ have suggested that dietary intervention strategies can result in long-term improvements in dietary intake, in some cases with parallel improvements in health $^{(14-16)}$ or reduced subsequent disease onset ${ }^{(17,18)}$. Success of such interventions may be improved by direct or indirect means of increasing exposure of individuals to specific foods/diets and continued counselling or follow-up. There are limited data demonstrating the impact of provision of foods within an intervention period on long-term dietary changes. In one previous report, it has been observed that $14 \mathrm{~d}$ exposure to a range of six vegetables significantly increased children's preference and intake of these vegetables 2 weeks post-intervention ${ }^{(19)}$, compared with either an information-only intervention or no intervention. Provision of free fruits for a school year to Norwegian children resulted in significant increases in fruit intake up to 3 years post-intervention $^{(20)}$. We are not aware of similar data available in the literature related to dietary interventions with whole-grain foods. The WHOLEheart study was a randomised, controlled trial testing the impact of increased consumption of wholegrain foods (wholemeal breads, breakfast cereals, pasta, rice and snacks were made available to participants) on markers of CVD risk in infrequent whole-grain consumers over a 16 -week period ${ }^{(21)}$. In brief, participants were recruited to

Abbreviation: WG, whole-grain intervention group.

*Corresponding author: Professor C. J. Seal, email chris.seal@ncl.ac.uk 
the present study based on age (18+ years), BMI in excess of $25 \mathrm{~kg} / \mathrm{m}^{2}$ and low baseline whole grain consumption equivalent to less than $20 \mathrm{~g}$ whole grains/d. This intervention provided a unique opportunity to assess habit forming associated with the dietary intake of whole-grain foods by comparing the subsequent intake of whole-grain foods in a group of infrequent whole-grain consumers who had been provided and consumed whole-grain foods over a 16-week period, with a comparator control group (who had made no prior dietary changes).

The aim of the present study was to compare whole-grain food intake in individuals who had previously consumed whole-grain foods as part of a whole-grain food intervention with a control group who made no such dietary change 1 year post-intervention. As a secondary aim, we compared whether there were changes in total dietary intake between the two participant groups over this time period.

\section{Methods}

\section{Participants}

All participants gave written informed consent. The present study was conducted according to the guidelines laid down in the Declaration of Helsinki. The study was approved by the North Tyneside Local Research Ethics Committee (reference $05 /$ Q0905/75) and by the Newcastle upon Tyne NHS Foundation Trust R\&D Department. The study was registered with isrtcn.org (SRCT no. CCT-NAPN-A13175).

The dietary intervention (the WHOLEheart study) from which this sample was drawn recruited 266 overweight, but otherwise healthy, individuals who ate less than one serving of whole grains daily (a serving defined as $20 \mathrm{~g}$ whole grains). Participants were randomly allocated into three groups: a control group who were asked to make no dietary changes over the 16-week intervention period; intervention group 1 (provided with whole-grain foods, and asked to consume three servings/d over the 16-week period); intervention group 2 (provided with whole-grain foods, and asked to consume three $20 \mathrm{~g}$ servings/d for 8 weeks, followed by six servings of whole grains/d for the subsequent 8 weeks). Full details of participant recruitment and intervention design for the WHOLEheart study have been described elsewhere ${ }^{(21)}$.

Upon completion of the intervention, participants were asked whether they would be willing to take part in further research activities. Between 48 and $49 \%$ of control participants and 41 and $52 \%$ of intervention participants provided FFQ data at the post-intervention time points.

\section{Collection of dietary data}

Positive respondents were sent FFQ to return by post at 1,6 and 12 months post-intervention. The FFQ, which recorded food intake only during the previous $7 \mathrm{~d}$, was the same as that used in the WHOLEheart intervention. The 149-item questionnaire was based on the UK European Prospective Investigation into Cancer and Nutrition FFQ, expanded to include inputs for both whole-grain foods provided for the study and other whole-grain foods available in the UK at the time of the study. Within the WHOLEheart intervention, nutrient intake determined from the FFQ was highly correlated with similar estimates from parallel food diaries ${ }^{(22)}$.

\section{Analysis of dietary data}

Whole grain consumption was assessed from returned FFQ data, using estimates of portion size based on previously reported average male and female whole-grain food portion sizes ${ }^{(10)}$. Participants' nutrient profile for total dietary intake was estimated using National Diet and Nutrition Survey 2000-1 $1^{(23)}$ data on portion sizes, and estimates of the nutrient content of a range of food items (McCance \& Widdowson ${ }^{(24)}$ data). All data were collated within a custom-made dietary database (Microsoft Access; Microsoft Office).

\section{Statistical analysis}

Dietary intake of participants who received whole grains during the intervention (WG group) and those who did not (control group) were compared at each time point, separately and when aggregated across the whole 12 months by the two-sample Wilcoxon rank-sum test using the Stata version 10.1 software package (StataCorp LP). A $P$ value of less than 0.05 was considered as statistically significant. For the purpose of the present analysis, post-intervention food intake data for WG groups 1 and 2 were combined to compare those who were provided with whole-grain foods during the intervention with those who did not.

\section{Results}

Return rates for FFQ were 55, 53 and $47 \%$ at 1-, 6- and 12 -month post-intervention time points, respectively (compared with $>90 \%$ return during the intervention). Data from the responders from each group are highlighted in Table 1. The mean post-intervention whole grain consumption over 12 months for each group is shown in Fig. 1. The demography of the respondents ( $52 \%$ females, with a median BMI of $29 \cdot 1 \mathrm{~kg} / \mathrm{m}^{2}$ and a median age of 47 years) was very similar to that of all participants in the WHOLEheart intervention $\left(50 \%\right.$ females, with a median BMI of $30.0 \mathrm{~kg} / \mathrm{m}^{2}$ and a median age of 46 years).

The mean whole grain intake in the control group was between 16 and $19 \mathrm{~g}$ across the four measurement periods and did not change from pre-intervention levels (Table 1). In the control group, $14-15 \%$ of the participants consumed no whole-grain foods at 1 and 6 months post-intervention; $25 \%$ consumed no whole-grain foods at 12 months postintervention. In contrast, only 3,7 and $6 \%$ of those from the WG group consumed no whole-grain foods at 1,6 and 12 months post-intervention. Whole grain consumption was significantly higher in intervention group 1 and intervention group 2 participants throughout the post-intervention period compared with both the control group and baseline $(P<0.001)$. However, whole grain consumption was not significantly different between the two intervention groups 
Table 1. Whole grain consumption in WHOLEheart volunteers 1, 6 and 12 months post-intervention*

(Mean values and standard deviations; number of subjects and percentages)

\begin{tabular}{|c|c|c|c|c|c|c|}
\hline & \multicolumn{3}{|c|}{ Control } & \multicolumn{3}{|c|}{$W G$} \\
\hline & $\begin{array}{c}1 \\
\text { month }\end{array}$ & $\begin{array}{c}6 \\
\text { months }\end{array}$ & $\begin{array}{c}12 \\
\text { months }\end{array}$ & $\begin{array}{c}1 \\
\text { month }\end{array}$ & $\begin{array}{c}6 \\
\text { months }\end{array}$ & $\begin{array}{c}12 \\
\text { months }\end{array}$ \\
\hline$n$ & 49 & 48 & 49 & 87 & 86 & 68 \\
\hline \multicolumn{7}{|l|}{$\begin{array}{l}\text { WG intake } \\
(\mathrm{g} / \mathrm{d})\end{array}$} \\
\hline Mean & $24 \cdot 3$ & 23.5 & $23 \cdot 8$ & $47 \cdot 1$ & 44.2 & $36 \cdot 3$ \\
\hline SD & $24 \cdot 2$ & $22 \cdot 2$ & $26 \cdot 7$ & 29.0 & 33.8 & $29 \cdot 3$ \\
\hline \multicolumn{7}{|l|}{$\begin{array}{l}\text { Non-WG } \\
\text { consumers }\end{array}$} \\
\hline$n$ & 7 & 7 & 12 & 3 & 6 & 4 \\
\hline$\%$ & $14 \cdot 2$ & 14.6 & 24.5 & 3.4 & 6.9 & 5.9 \\
\hline
\end{tabular}

WG, whole-grain intervention group.

${ }^{*}$ Approximately equal numbers of respondents came from the two WG in the original WHOLEheart study at each time point (intervention group $1 v$. intervention group 2: 1 month, $n 45$ v. 42; 6 months, $n 41$ v. 45; 12 months, $n 33$ v. 35).

during the post-intervention period, so the data are combined in Table 1 for ease of comparison. There was a decrease in whole grain consumption for the WG group between 1 month and 12 months post-intervention, but this was not statistically significant, and at the end of the measurement period, this group of subjects was consuming approximately $50 \%$ more whole grains than the control group.

Table 2 details the specific differences that occurred in terms of whole-grain food consumption during the intervention and over the 12-month post-intervention period.
The WG group participants had a significantly higher intake of whole-grain breads and breakfast cereals compared with the control group at each of the three time points assessed over the 12-month post-intervention period. The consumption of whole-grain pasta and rice, as well as whole-grain snacks was significantly higher in the WG group at some time points post-intervention ( 1 and 6 months for pasta/rice and 12 months for whole-grain snacks).

The frequency of consumption of specific food groups by participants at each time point is shown in Table 3. Participation in the intervention arms of the WHOLEheart intervention resulted in a significantly higher daily frequency of breakfast cereal consumption (overall $P=0.002$ ) at 1,6 and 12 months post-intervention. The average increase in the daily frequency of breakfast cereal consumption was about 0.30 occasions/d compared with the values between 0.43 and 0.57 occasions/d seen during the intervention when whole-grain breakfast cereals were provided to the participants. Overall, across the 12-month follow-up period, the frequency of snack consumption was lower in those participants from the WG group compared with the control group $(P=0 \cdot 020)$. Differences in the other food groups were small, with no consistent differences between the control and WG groups. Specifically, the frequency of bread consumption, which had been significantly higher in the WG group at the end of the 16-week intervention period, was not different between the WG and control groups throughout the post-intervention follow-up period. Daily energy intake was not different between the control and intervention group participants throughout the post-intervention period,

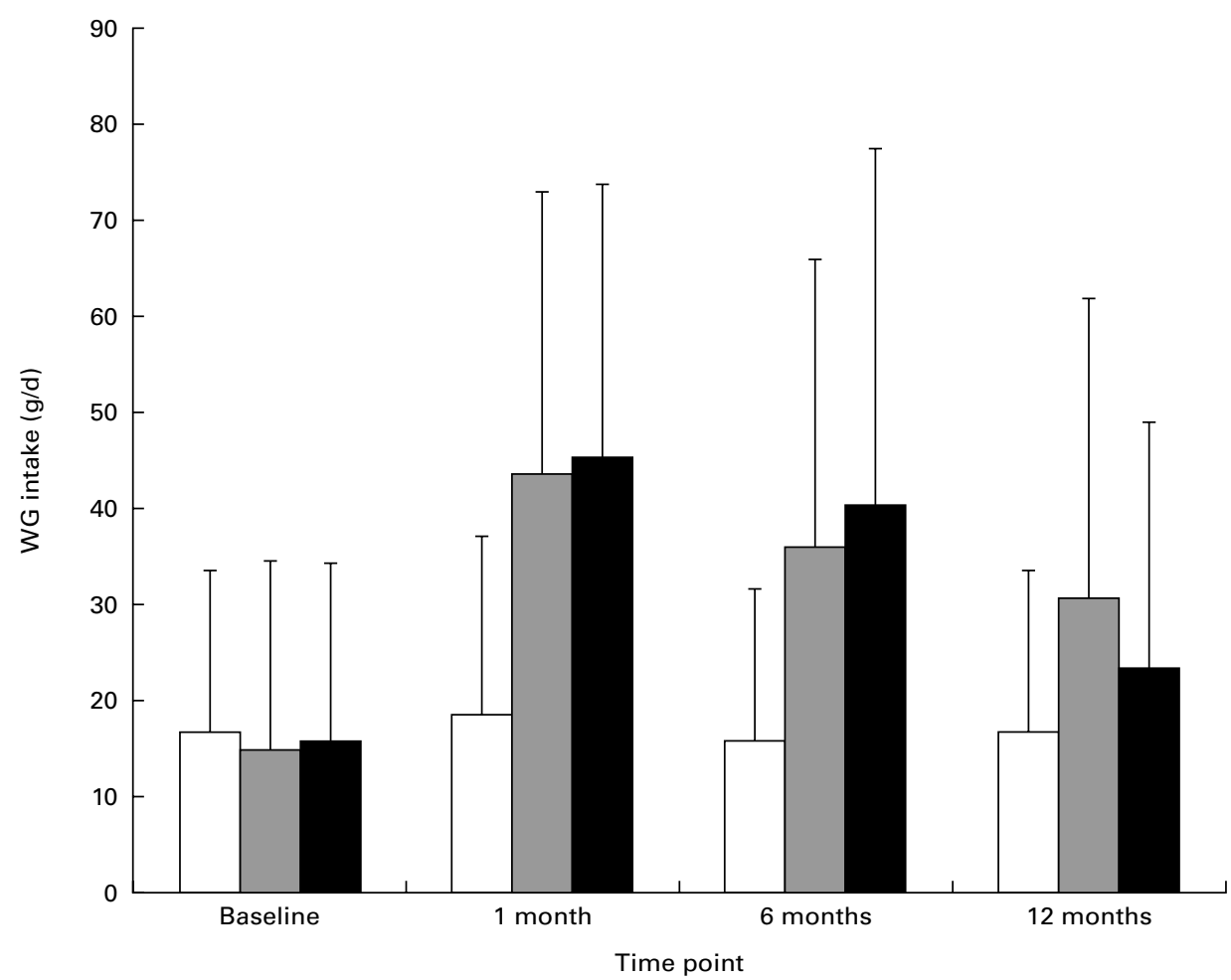

Fig. 1. Mean whole grain (WG) intake at baseline (pre-intervention) and post-intervention time points. $\square$, Control group; $\square$, intervention group 1 (prescribed $60 \mathrm{~g}$

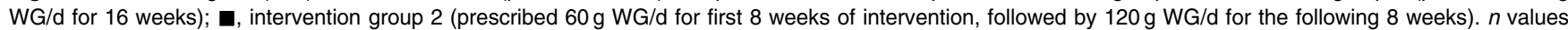
at baseline are 94 (control), 76 (intervention group 1) and 80 (intervention group 2) at 1 month. 
and was comparable with measures of energy intake recorded at baseline and during the intervention itself (data not shown, $P>0.350$ at all time points). An increase in estimated dietary fibre intake seen during the intervention in those consuming whole-grain foods continued into the post-intervention period ( $P<0.001$ overall across 1, 6 and 12 months), although the differences were smaller and only significant at the 12-month time point (mean for control $v$. intervention groups: 16.5 (SD 5.3) v. 19.8 (SD 7.3) g/d, $P=0.004$ ). Other differences between the control and WG groups seen during the intervention itself (reductions in the proportion of fat and corresponding increase in carbohydrate and increases in some micronutrients) were not consistently different during the follow-up.

\section{Discussion}

This follow-up evaluation suggests that a relatively short, direct dietary intervention with whole-grain foods can have a significant impact on long-term whole grain consumption in individuals who habitually consume low amounts of such foods. The WG group maintained a higher intake of whole grains ( $>10 \mathrm{~g} / \mathrm{d}$ compared with the control group) over the 12 -month period. It appears as though both an increased percentage of non-consumers of whole grains (at 6 and 12 months post-intervention) and a reduction in elective whole grain consumption cause a gradual reduction in whole grain consumption over time, but this was not statistically significant. This is consistent with evidence that repeated 'exposure' to foods that children spontaneously elect not to consume can increase subsequent, long-term dietary inclusion $^{(25)}$. Data from our control group suggest that habitual whole grain consumption is still much lower than that recommended in the USA or Denmark. A number of reasons have been suggested previously as to why individuals in the UK choose refined grain options as opposed to whole grains ${ }^{(26)}$. However, only a small percentage of the participants withdrew from the WHOLEheart intervention period citing unwillingness to consume whole-grain foods as their reason for withdrawal. This suggests that taste acceptability of wholegrain products is possibly higher than the expected taste of these products once the participants had been exposed to them. Qualitative research undertaken with the WHOLEheart volunteers suggests that there are personal, product-related or situational factors that prevent habitual inclusion of whole-grain foods in the diet ${ }^{(27)}$.

The most striking effects on dietary habits were the significant increase in the frequency of breakfast cereal consumption at all post-intervention time points (see Table 3), and the significant increase in NSP intake in the WG group $v$. the control group when all time points were considered. Increased frequency of breakfast consumption has been linked to health benefits in population-based studies ${ }^{(28,29)}$, emphasising the additional benefit of this change in dietary pattern. Otherwise, there was little impact on the frequency of consumption of other foods when compared with the intake of the control group.

The 'gold standard' for the estimation of dietary intake is through the use of food diaries ${ }^{(30,31)}$. However, participants 
are often less compliant when filling out diaries due to the increased time burden and inconvenience involved ${ }^{(30)}$. As a result, the FFQ was the method of choice in the present study for dietary data collection, in order to improve return rate from as many participants as possible. As this was the methodology for collection used during the WHOLEheart intervention period, its use was familiar to participants and provided data directly comparable with previously collected data.

The average consumption of whole-grain foods across the 12-month post-intervention follow-up period, although not statistically significant, appeared to decline, suggesting a gradual removal of whole-grain foods from the diets of those from the WG group. The mean intakes observed fell short of intakes recommended in the USA of $48 \mathrm{~g}$ whole grains $/ \mathrm{d}^{(8)}$, and the more recent Danish recommendations of $75 \mathrm{~g}$ whole grains $/ \mathrm{d}^{(9)}$. However, the mean levels of whole grain consumption measured after 12 months post-intervention (over $36 \mathrm{~g} / \mathrm{d}$ ) are considerably higher than the most recent estimates of whole grain intake in adults both in the UK (median of $14 \mathrm{~g} / \mathrm{d}$ from $2000-1$ data $^{(10)}$ ) and the USA (mean values of approximately $11 \mathrm{~g} / \mathrm{d}$ from $1999-2004$ data $^{(32)}$ ).

The length of the whole-grain food intervention within the present study was longer than any previous whole-grain intervention ( 4 months). As such, there is no indication from the present data whether a shorter intervention with whole-grain foods would also result in longer-term, elective whole grain consumption. Specific strategies within this intervention period, and perhaps the method of dietary intervention ${ }^{(33)}$, may also increase the time and amplitude of post-intervention elective whole-grain food consumption. A range of further consumer studies are required to optimise the time, length, magnitude and mode of such interventions. Such studies in long-term, post-intervention dietary changes are not only important to the development of public health messages aimed at increasing the intake of whole grains or other foods, but may also allow for more effective food marketing strategies to further increase the chances of measurable public health benefits.

The participants in the present study were specifically chosen because of their low consumption of whole-grain foods at recruitment. They were provided with a range of whole-grain foods to include in their diet during the intervention period. However, subsequent increases in whole grain consumption (compared with baseline or the control group) represent an increase in the elective consumption of wholegrain foods by these participants. This increased intake was maintained over the course of a 12-month period. As such, dietary intervention with whole-grain foods may represent a strategy through which health professionals (e.g. through provision of sample whole-grain food packs to target groups) or food industry professionals (e.g. through promotions or free samples) could increase the intake of whole-grain food products in the general population. As suggested above, the increased whole grain consumption noted here would not result in the lowest whole-grain consumers reaching dietary guideline levels. However, it would bring such individuals closer to these goals, and aid them in maintaining a higher whole grain intake over a number of months. 


\section{Acknowledgements}

The present study was supported by the UK Food Standards Agency (project N02036). The authors designed the study; I. A. B. and S. A. K. managed the participant data collection; I. A. B. analysed the dietary intake data. All authors were involved in the data interpretation and manuscript preparation. The authors report no conflicts of interest arising from the present research or its publication.

\section{References}

1. Jacobs DR, Slavin J \& Marquart L (1995) Whole grain intake and cancer - a review of the literature. Nutr Cancer Int J $\mathbf{2 4}$, 221-229

2. Jacobs DR, Meyer KA, Kushi LH, et al. (1999) Is whole grain intake associated with reduced total and cause-specific death rates in older women? The Iowa Women's Health Study. Am J Public Health 89, 322-329.

3. Anderson JW (2003) Whole grains protect against atherosclerotic cardiovascular disease. Proc Nutr Soc 62, 135-142.

4. Steffen LM, Jacobs DR, Stevens J, et al. (2003) Associations of whole-grain, refined-grain, and fruit and vegetable consumption with risks of all-cause mortality and incident coronary artery disease and ischemic stroke: the Atherosclerosis Risk in Communities (ARIC) Study. Am J Clin Nutr 78, 383-390.

5. Mancino L, Kuchler F \& Leibtag E (2008) Getting consumers to eat more whole-grains: the role of policy, information, and food manufacturers. Food Policy 33, 489-496.

6. Good CK, Holschuh N, Albertson AM, et al. (2008) Whole grain consumption and body mass index in adult women: an analysis of NHANES 1999-2000 and the USDA pyramid servings database. J Am Coll Nutr 27, 80-87.

7. Kyrø C, Skeie G, Dragsted LO, et al. (2012) Intake of whole grain in Scandinavia: intake, sources and compliance with new national recommendations. Scand J Public Health $\mathbf{4 0}$, $76-84$.

8. US Department of Agriculture (2005) MyPlate. http://www. choosemyplate.gov/ (accessed October 2011).

9. The Danish Wholegrain Campaign (2009) Vaelg Fuldkorn Forst (Choose wholegrain first). http://www.fuldkorn.dk/ index.php?pageid=15 (accessed October 2011).

10. Thane CW, Jones AR, Stephen AM, et al. (2007) Comparative whole-grain intake of British adults in 1986-7 and 2000-1. Br J Nutr 97, 987-992.

11. Bradbury J, Thomason JM, Jepson NJA, et al. (2006) Nutrition counseling increases fruit and vegetable intake in the edentulous. J Dent Res 85, 463-468.

12. Takahashi Y, Sasaki S, Okubo S, et al. (2006) Maintenance of a low-sodium, high-carotene and -vitamin $\mathrm{C}$ diet after a 1-year dietary intervention: The Hiraka Dietary Intervention Follow-up Study. Preven Med 43, 14-19.

13. Wrieden WL, Anderson AS, Longbottom PJ, et al. (2007) The impact of a community-based food skills intervention on cooking confidence, food preparation methods and dietary choices - an exploratory trial. Public Health Nutr 10, 203-211.

14. Esposito K, Marfella R, Ciotola M, et al. (2004) Effect of a Mediterranean-style diet on endothelial dysfunction and markers of vascular inflammation in the metabolic syndrome: a randomized trial. JAMA 292, 1440-1446.

15. Charlton KE, Steyn K, Levitt NS, et al. (2008) A food-based dietary strategy lowers blood pressure in a low socio-econ- omic setting: a randomised study in South Africa. Public Health Nutr 11, 1397-1406.

16. Shai I, Schwarzfuchs D, Henkin Y, et al. (2008) Weight loss with a low-carbohydrate, Mediterranean, or low-fat diet. $N$ Engl J Med 359, 229-241.

17. Lindstrom J, Ilanne-Parikka P, Peltonen M, et al. (2006) Sustained reduction in the incidence of type 2 diabetes by lifestyle intervention: follow-up of the Finnish Diabetes Prevention Study. Lancet 368, 1673-1679.

18. Cook NR, Cutler JA, Obarzanek E, et al. (2007) Long term effects of dietary sodium reduction on cardiovascular disease outcomes: observational follow-up of the trials of hypertension prevention (TOHP). BMJ 334, 885-888.

19. Wardle J, Cooke LJ, Gibson EL, et al. (2003) Increasing children's acceptance of vegetables; a randomized trial of parent-led exposure. Appetite 40, 155-162.

20. Bere E, Veierod MB, Bjelland M, et al. (2006) Free school fruit - sustained effect 1 year later. Health Educ Res 21, $268-275$.

21. Brownlee IA, Moore C, Chatfield M, et al. (2010) Markers of cardiovascular risk are not changed by increased wholegrain intake: the WHOLEheart study, a randomised, controlled dietary intervention. Br J Nutr 104, 125-134.

22. Brownlee IA, Kuznesof SA \& Chatfield M, et al. (2008) Randomised Controlled Trial to Test the Impact of Increased Consumption of Wholegrain Foods on Cardiovascular Disease Risk (The WHOLEheart Study): Final Report to the Food Standards Agency. London: Food Standards Agency.

23. UK Food Standards Agency (2005) SN 5140 - National Diet and Nutrition Survey: Adults Aged 19 to 64 Years, 2000-2001. London: Food Standards Agency.

24. McCance RA \& Widdowson EM (2001) McCance and Widdowson's The Composition of Foods, Gth ed. Cambridge: Royal Society of Chemistry.

25. Birch LL \& Wolfe MD (1982) I don't like it; I never tried it: effects of exposure on two-year-old children's food preferences. Appetite 3, 353-360.

26. Smith AT, Kuznesof S, Richardson DP, et al. (2003) Behavioural, attitudinal and dietary responses to the consumption of wholegrain foods. Proc Nutr Soc 62, 455-467.

27. Kuznesof S, Brownlee IA, Moore C, et al. (2012) WHOLEheart study participant acceptance of wholegrain foods. Appetite 59, 187-193.

28. Keski-Rahkonen A, Kaprio J, Rissanen A, et al. (2003) Breakfast skipping and health-compromising behaviors in adolescents and adults. Eur J Clin Nutr 57, 842-853.

29. Mota J, Fidalgo F, Silva R, et al. (2008) Relationships between physical activity, obesity and meal frequency in adolescents. Ann Hum Biol 35, 1-10.

30. Bingham SA, Cassidy A, Cole TJ, et al. (1995) Validation of weighed records and other methods of dietary assessment using the $24 \mathrm{~h}$ urine nitrogen technique and other biological markers. Br J Nutr 73, 531-550.

31. Bingham SA, Gill C, Welch A, et al. (1997) Validation of dietary assessment methods in the UK arm of EPIC using weighed records, and 24-hour urinary nitrogen and potassium and serum vitamin $\mathrm{C}$ and carotenoids as biomarkers. Int J Epidemiol 26, S137-S151.

32. O'Neil CE, Zanovec M, Cho SS, et al. (2010) Whole grain and fiber consumption are associated with lower body weight measures in US adults: National Health and Nutrition Examination Survey 1999-2004. Nutr Res 30, 815-822.

33. Rosen RA, Schmitz K, Cohen EA, et al. (2009) A gradual approach to increasing whole grain consumption in children. Cereal Foods World 54, 113-117. 